\title{
Probing Fundamental Symmetries of Deformed Nuclei in Symmetric Top Molecules
}

\author{
Phelan Yu $\odot^{*}$ and Nicholas R. Hutzler $\odot^{\dagger}$ \\ Division of Physics, Mathematics, and Astronomy, California Institute of Technology, Pasadena, California 91125, USA
}

(Received 21 August 2020; accepted 24 November 2020; published 11 January 2021)

\begin{abstract}
Precision measurements of Schiff moments in heavy, deformed nuclei are sensitive probes of beyond standard model $T, P$ violation in the hadronic sector. While the most stringent limits on Schiff moments to date are set with diamagnetic atoms, polar polyatomic molecules can offer higher sensitivities with unique experimental advantages. In particular, symmetric top molecular ions possess $K$ doublets of opposite parity with especially small splittings, leading to full polarization at low fields, internal comagnetometer states useful for rejection of systematic effects, and the ability to perform sensitive searches for $T, P$ violation using a small number of trapped ions containing heavy exotic nuclei. We consider the symmetric top cation ${ }^{225} \mathrm{RaOCH}_{3}^{+}$as a prototypical and candidate platform for performing sensitive nuclear Schiff measurements and characterize in detail its internal structure using relativistic ab initio methods. The combination of enhancements from a deformed nucleus, large polarizability, and unique molecular structure make this molecule a promising platform to search for fundamental symmetry violation even with a single trapped ion.
\end{abstract}

DOI: $10.1103 /$ PhysRevLett.126.023003

Searches for permanent electric dipole moments (EDMs) in atoms and molecules are powerful probes of time reversal and parity $(T, P-)$ violating physics posited by beyond standard model (BSM) theories [1,2]. Unsuppressed $T, P$ violation, and by extension charge conjugation-parity $(C P)$ violation, are needed to explain the observed lack of free antimatter in the Universe [3]. In the standard model, however, $C P$ violation only weakly manifests in quark and neutrino mixing phases and is apparently absent for strong interactions (the strong $C P$ puzzle) [4]. The hadronic sector thus provides a natural venue for introducing many new $C P$-violating BSM interactions to resolve this discrepancy [5-7].

New $T, P$-violating nuclear effects, including nucleonnucleon interactions mediated by QCD, are understood to induce a collective EDM in atomic nuclei of nonzero spin known as a Schiff moment [8-10]. This effect, which scales with atomic mass $Z$, is particularly pronounced in heavy, octopole-deformed nuclei, such as ${ }^{225} \mathrm{Ra}[11,12]$, where low-lying nuclear states couple strongly to the oppositeparity ground state [13]. The resulting Schiff moment, and corresponding sensitivity to BSM physics, is a factor of ¿100 larger [14-16] when compared to heavy spherical nuclei, such as ${ }^{129} \mathrm{Xe}[17,18]$ and ${ }^{199} \mathrm{Hg}$ [19], the latter of which is used in the current most sensitive Schiff moment experiment.

Heavy, octopole-deformed isotopes, however, are typically short-lived and difficult to produce in large quantities $[11,13,20]$. Maximizing experimental sensitivity and coherence time is thus paramount to overcoming a limited count rate. One demonstrated method for increasing experimental sensitivity is to use a polar molecule, whose internal fields can be easily oriented to provide an enhancement of $\gtrsim 100$ over atoms in EDM measurements [21-23]. TlF, for instance, is sensitive to the Schiff moment of ${ }^{205} \mathrm{Tl}$ nuclei [24-26], and theoretical proposals have identified a wide variety of diatomic [27-33] and triatomic molecules [28,34,35] suitable for Schiff moment measurements. Combining enhancements due to nuclear deformation and the polarizability of molecules results in $\gtrsim 10^{5}$ sensitivity increase relative to atomic Schiff moment measurements with spherical nuclei.

Molecular ions have proven to be a powerful platform for very sensitive measurements of symmetry violation [22] due to long trapping and coherence times [36]. This enables the ability to perform measurements with small quantities of the target molecule, for example, those containing scarce or unstable nuclei. However, many BSM-sensitive species, including radium, do not have the prerequisite electronic structure to make diatomic molecular ions with oppositeparity $(\Omega)$ doublets, which are needed to fully realize the advantages of this approach. Polyatomic molecules, by contrast, possess rovibrational parity doublets [34] and thus provide a generic approach to conducting molecular ion measurements with a broad range of useful, and possibly rare, species.

In this Letter, we consider symmetric top molecular (STM) ions as a new platform for Schiff moment measurements. Our proposal improves upon contemporary probes of hadronic $C P$ violation by combining nuclear and molecular enhancements with the advantages of polyatomic structure and extended ion trap coherence times-features which will also benefit a variety of other precision measurements. A prototypical molecule for our 
approach, the radium monomethoxide cation $\left(\mathrm{RaOCH}_{3}^{+}\right)$, was recently synthesized and cotrapped [37] with lasercooled $\mathrm{Ra}^{+}$[38]. The axial symmetry of this molecule gives rise to near-degenerate opposite parity $K$ doublets, thereby enabling full polarization in small fields and the comagnetometer states necessary for precision measurements in an ion trap. The ground electronic state $\left(\tilde{X}^{1} A_{1}\right)$ is diamagnetic, suppressing sensitivity to magnetic noise. Because of this combination of enhancements and features, even a single trapped $\mathrm{RaOCH}_{3}^{+}$ion could be used to explore interesting parameter space for new physics.

There are several advantages to using a more complex STM ion, as opposed to a triatomic analog (e.g., $\mathrm{RaOH}^{+}$) $[28,34,39]$. First, the increased rovibrational complexity of an STM, which makes laser cooling of neutral species more difficult (though indeed possible [40,41]), does not pose challenges for the control of STM ions, as trapped ions do not require photon cycling to achieve high precision $[22,36]$. Furthermore, $K$ doublets, which arise from rotational degrees of freedom, can arise in any vibrational state, such as the ground state considered here. They are therefore low-lying $(\nu \sim 100 \mathrm{GHz})$, have vastly longer radiative lifetimes than excited vibrational modes, and possess smaller splittings than the $\ell$ doublets of triatomics.

Our theoretical analysis focuses on ${ }^{225} \mathrm{RaOCH}_{3}^{+}$, which contains the short-lived $\left(\tau_{1 / 2} \approx 15 \mathrm{~d}\right)$ spin- $1 / 2$ radium isotope. We examine in detail the ground state hyperfine structure, as well as the various contributions to the degeneracy breaking of the $K$ states. We furthermore identify states suitable for measurement of a Schiff moment, including comagnetometer states, and examine the Stark and Zeeman effects in the molecule.

Internal structure and $K$ doubling.-The internal structure of the electronic ground state $\left(\tilde{X}^{1} A_{1}\right)$ is analyzed with explicit diagonalization of the effective molecular Hamiltonian: $H_{\text {total }}=H_{\text {rot }}+H_{\text {Stark }}+H_{\text {Zeeman }}+H_{\text {ss }}+H_{\text {nsr }}+H_{\text {sm }}$. We have included the rotational (rot), Stark, Zeeman, nuclear spin dipolar (ss), and nuclear spin-rotation terms (nsr), all of which are generic to STMs. The Schiff moment (sm) term arises from the ${ }^{225} \mathrm{Ra}(I=1 / 2)$ nucleus, and is $T$, $P$ violating. Similar to the closed-shell alkali monomethyls [42], electron spin terms are omitted. We obtain molecular parameters (see Table I) using a variety of relativistic $a b$ initio methods. Geometries are optimized at the level of $\operatorname{CCSD}(\mathrm{T})$ with an ANO-RCC-VQZ basis [43-48] via CFOUR [49-51], and scalar relativistic effects are modeled using the one-electron variant of spin-free $\mathrm{X} 2 \mathrm{C}$ theory [52-54]. Nuclear spin-rotation and rotational Zeeman parameters are computed via a four-component relativistic linear response approach [55-57] in the DIRAC19 code [58] using the dyall.v4z basis [59], and electron correlation is treated at the DFT level with a B3LYP functional [60]. Additional details on the derivation of the Hamiltonian and $a b$ initio parameters can be found in the Supplemental Material [61].
TABLE I. Molecular parameters for ${ }^{225} \mathrm{RaOCH}_{3}^{+}$ground state $\left(\tilde{X}^{1} A_{1}\right)$. See Supplemental Material [61] for details.

\begin{tabular}{|c|c|c|c|}
\hline \multicolumn{2}{|l|}{ Hyperfine } & $T_{z z}$ & $\left|T_{x x}-T_{y y}\right|$ \\
\hline \multirow{3}{*}{\multicolumn{2}{|c|}{$\begin{array}{l}T\left[C_{\mathrm{nsr}}\left({ }^{225} \mathrm{Ra}\right)\right] \\
T\left[C_{\mathrm{nsr}}\left({ }^{1} \mathrm{H}\right)\right] \\
\alpha_{\text {dip }} T\left[C_{\text {dip }}\left({ }^{225} \mathrm{Ra}-\mathrm{H}\right)\right]^{\mathrm{a}}\end{array}$}} & $3.67 \mathrm{kHz}$ & \\
\hline & & $15.3 \mathrm{kHz}$ & $0.301 \mathrm{kHz}$ \\
\hline & & $-38.0 \mathrm{kHz}$ & $0.391 \mathrm{kHz}$ \\
\hline \multicolumn{2}{|l|}{ Geometry } & \multicolumn{2}{|c|}{ Stark and Zeeman } \\
\hline $\mathrm{r}(\mathrm{Ra}-\mathrm{O})$ & $2.1949 \AA$ & $d_{0}$ & $4.969 \mathrm{D}$ \\
\hline $\mathrm{r}(\mathrm{O}-\mathrm{C})$ & $1.4076 \AA$ & $g_{N}\left({ }^{225} \mathrm{Ra}\right)^{[84]}$ & $-0.7338 \mu_{N}$ \\
\hline $\mathrm{r}(\mathrm{C}-\mathrm{H})$ & $1.0864 \AA$ & $g_{N}(\mathrm{H})^{[85]}$ & $2.7928 \mu_{N}$ \\
\hline$\angle(\mathrm{O}-\mathrm{C}-\mathrm{H})$ & $110.73^{\circ}$ & $g_{R(\|)}$ & $6.65 \mu_{N}$ \\
\hline$\angle(\mathrm{H}-\mathrm{C}-\mathrm{H})$ & $108.18^{\circ}$ & $g_{R(\perp)}$ & $0.619 \mu_{N}$ \\
\hline$A$ & $5.4010 \mathrm{~cm}^{-1}$ & & \\
\hline$B$ & $0.0673 \mathrm{~cm}^{-1}$ & & \\
\hline
\end{tabular}

${ }^{a}$ The scaling constant for the dipolar spin-spin interaction is defined as $\alpha_{\text {dip }}=-\sqrt{6} \mu_{0} \gamma_{\mathrm{H}} \gamma_{\mathrm{Ra}} \hbar^{2} / 4 \pi$.

The rotational structure of symmetric tops is parametrized by three quantum numbers: the electronic angular momentum apart from spin $(N)$, its molecule-frame projection $(K)$, and its lab-frame projection $\left(m_{N}\right)$. For $|K|>0$, which corresponds to rotation about the symmetry axis, the cylindrical symmetry of the molecule gives rise to a pair of degenerate $+K$ and $-K$ states within each $|N| K|\rangle$, rotational manifold (see Fig. 1). These degeneracies can be lifted by hyperfine and centrifugal terms that couple states of different $K$, leading to the formation of near-degenerate opposite parity $K$ doublets, $| \pm\rangle=(|N,+K\rangle \pm|N,-K\rangle) / \sqrt{2}$.

We propose to use the $N=|K|=1$ manifold for the Schiff moment search. This state is $\sim 160 \mathrm{GHz}$ above the

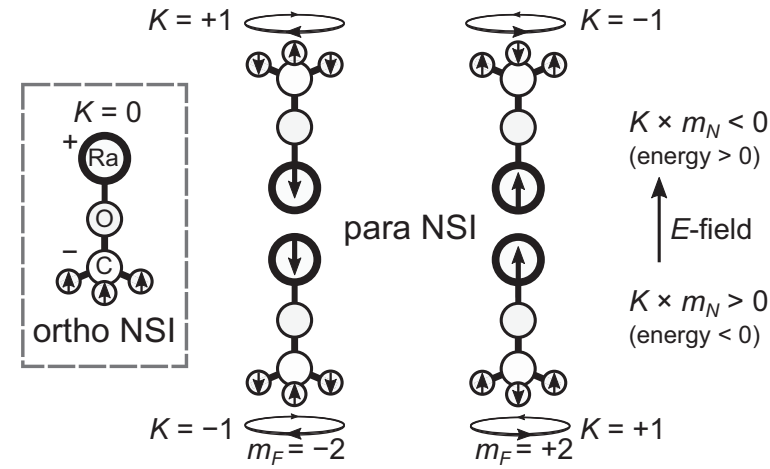

FIG. 1. Labeled ${ }^{225} \mathrm{RaOCH}_{3}^{+}$STMs in stretched states, grouped by energy and total angular momentum projection $m_{F}$. The internuclear axis runs from the negatively charged end (methyl group) to the positively charged metal (radium). $K$ is the molecule-frame projection of angular momentum without spin $N$, while $m_{N}$ is its projection onto the lab frame. Nuclear spins for each spin-1/2 nucleus $\left({ }^{1} \mathrm{H},{ }^{225} \mathrm{Ra}\right)$ are indicated. The $|K|=1$ states correspond to the mixed para nuclear spin isomer (NSI) of the hydrogens, while the $K=0$ state coincides with the stretched ortho NSI. 


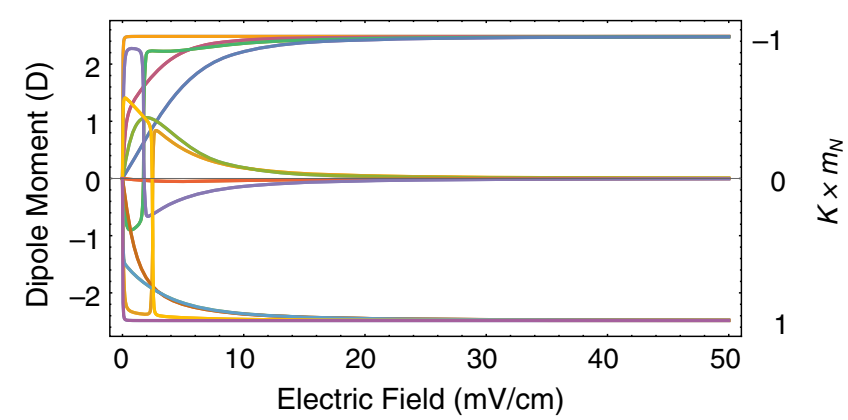

FIG. 2. Lab frame dipole moment of hyperfine states of the $|N=1| K,|=1\rangle$ manifold in the limit where the $K$ doublets are fully mixed yet rotational mixing is negligible. High, low, and no field seekers correspond to states with negative, positive, and zero dipole moment $\left(K \times m_{N}=+1,0\right.$, and -1$)$. The jumps indicate avoided crossings.

absolute ground state, and accordingly has a much longer lifetime than any vibrationally excited mode (with frequencies $\gtrsim 1 \mathrm{THz}$ ) such as those in triatomics. The spontaneous decay rate is further suppressed as the transition to the lower $K=0$ ground state is spin forbidden, making the radiative lifetime much longer than $1 \mathrm{~h}$ or any other relevant experimental timescale.

Similar to $\Omega$ and $\ell$ doublets, opposite parity $K$ doublets can be mixed in electric fields where the Stark energy exceeds the zero-field $K$-doublet splitting. In this regime, the molecule is polarized and its internal fields are oriented in the lab frame (see Fig. 2). This gives insensitivity to electric field fluctuations by largely saturating the Schiff moment sensitivity, as well as enabling comagnetometer states. In open-shell species, such as $\mathrm{CaOCH}_{3}$, the splitting between the ground state $K$ doublets is $\sim 0.3 \mathrm{MHz}$ [87], dominated by an anisotropic hyperfine interaction between the proton spins and the metal-centered electron. In contrast, the absence of unpaired electron spin in the ${ }^{225} \mathrm{RaOCH}_{3}^{+}$ground state implies that the dominant hyperfine contributions to $K$ doubling are from nuclear spin interactions, which are suppressed generically by at least an order of magnitude due to the minute size of nuclear magnetic moments compared to electronic magnetic moments $[88,89]$.

For the $N=|K|=1$ manifold, we calculate that anisotropic nuclear spin-spin and spin-rotation contributions from the hydrogen nuclei generate sub-kHz $K$ doublings. Combined with the calculated dipole moment of $\approx 5 \mathrm{D}$, this results in an extremely low threshold for reaching the highfield limit and polarizing the molecule. Indeed, we find that states in this manifold are $>90 \%$ polarized in external electric fields of $50 \mathrm{mVcm}^{-1}$ and $>99.9 \%$ polarized in fields of $250 \mathrm{mVcm}^{-1}$. This threshold is even lower for stretched states with maximal projection of total angular momentum $\left(m_{F}\right)$, which reach full polarization $(>99.9 \%)$ in fields as low as $\lesssim \mathrm{mV} \mathrm{cm}^{-1}$ (see Fig. 2), small enough that the molecules could be polarized in $\sim \mathrm{mK}$ deep optical traps [90]. Rotational mixing can be neglected at these small fields.

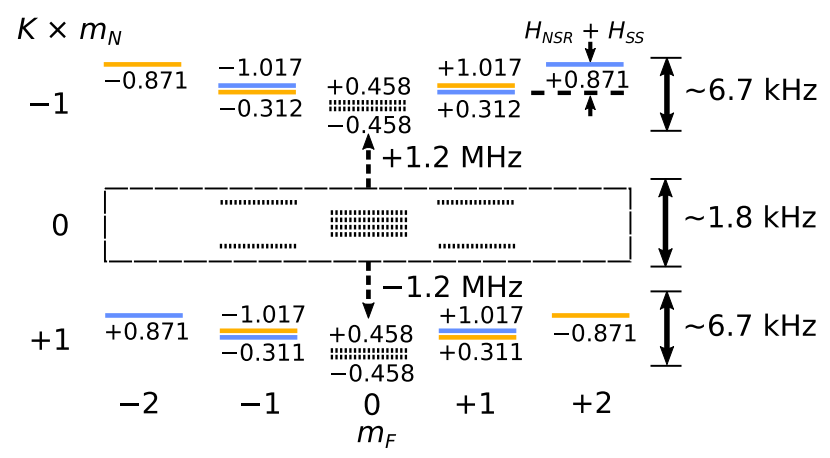

FIG. 3. Level structure and Schiff moment sensitivities for the 24 hyperfine states of the $N=|K|=1$ manifold in the decoupled regime $(1 \mathrm{~V} / \mathrm{cm})$, grouped by $m_{F}$ and their Stark manifold $\left(K \times m_{N}\right)$. Gold states have $+1 / 4$ effective Schiff sensitivity, while blue states have $-1 / 4$ effective Schiff sensitivity. Dashed lines denote zero Schiff sensitivity. Labels above or below the states indicate the effective $g$ factor at zero magnetic field, in terms of nuclear magnetons $\left(\mu_{N}\right)$. Table S4 in Ref. [61] lists the admixtures for each state.

The absence of unpaired electron spin in the $\tilde{X}^{1} A_{1}$ ground state also has implications for the magnetic level structure, as only the nuclear and rotational moments contribute to the Zeeman energy of the ground state. This results in suppressed sensitivity to magnetic fields and effective $g$ factors that are on the order of the nuclear magneton $\mu_{N}$ (see Fig. 3).

Hyperfine structure.-The large number of internal degrees of freedom in ${ }^{225} \mathrm{RaOCH}_{3}^{+}$necessitates a detailed treatment of the hyperfine structure to both identify resolvable states for the Schiff moment measurement as well as elucidate the sources of $K$ doubling. We use a fully decoupled basis, $\left|N, K, m_{N}\right\rangle\left|\Gamma, I_{H}, m_{I H}\right\rangle\left|I_{M}, m_{I M}\right\rangle$, to describe the hyperfine structure of the $\tilde{X}^{1} A_{1}$ electronic state. The spin of the metal ${ }^{225} \mathrm{Ra}$ nucleus is denoted $I_{M}$, and $m_{I M}$ is its corresponding lab frame projection. Similarly, the total nuclear spin of the three hydrogen atoms and its lab frame projection are denoted $I_{H}$ and $m_{I H}$, while $\Gamma$ denotes the symmetry character of the hydrogen spin wave function under $C_{3 v}$ transformations. In concrete terms, these hydrogen spin wave functions correspond to either of two nuclear spin isomers (NSIs): the "ortho" stretched states $\left(\Gamma=A, I_{H}=3 / 2\right)$ and the "para" mixed states $\left(\Gamma=E, I_{H}=1 / 2\right)$.

In each $|N| K|\rangle$, manifold, symmetry arguments restrict the allowed NSIs [91]. In particular, ortho states are only allowed with $K=3 n$ rotational states (for integer $n$ ), while the para states are associated with $K \neq 3 n$ states. All other combinations are forbidden by quantum statistics and $C_{3 v}$ symmetries. (See Supplemental Material [61] for details.) Accounting for these restrictions results in a total of 24 hyperfine states in the $N=1,|K|=1$ manifold, which are all resolvable in high fields.

Two types of hyperfine terms are present in the $\tilde{X}^{1} A_{1}$ state: dipolar couplings between the spins of different 
nuclei, and couplings between the nuclear spin and molecular rotation. The Hamiltonian for dipolar nuclear spin-spin interaction between two spins $\mathbf{I}_{1}$ and $\mathbf{I}_{2}$ can be expressed in terms of spherical tensors [92],

$$
H_{\mathrm{ss}}=-\sqrt{6} \frac{\mu_{0} \gamma_{1} \gamma_{2} \hbar^{2}}{4 \pi} T^{2}\left(C_{\mathrm{dip}}\right) \cdot T^{2}\left(\mathbf{I}_{1}, \mathbf{I}_{2}\right),
$$

where $\mu_{0}$ is the vacuum permeability, $\gamma_{1}, \gamma_{2}$ are the gyromagnetic ratios, and $C_{\text {dip }}$ is a spin-spin coupling tensor. For the $N=|K|=1$ manifold, we only need to consider spin-spin interactions between the ortho-NSI hydrogens and the ${ }^{225} \mathrm{Ra}$ atoms, as the interhydrogen matrix elements vanish between para-NSI states $[93,94]$. The dipolar spin coupling tensor $T^{2}\left(C_{\text {dip }}\right)$, which can be directly evaluated as a sum of spherical harmonics, gives a diagonal shift of $\sim 40 \mathrm{kHz}$ for $\mathbf{I}_{H} \cdot \mathbf{I}_{R}$ interactions. Anistropic couplings of $\sim 400 \mathrm{~Hz}$ mix states differing by $\Delta K=2$, which contributes to the $K$ doubling.

Nuclear spin rotation is the interaction between a nuclear magnetic moment associated with a spin $\mathbf{I}$ and the magnetic field created by the rotational angular momentum $\mathbf{N}[95]$,

$H_{\mathrm{nsr}}=\frac{1}{2} \sum_{k=0}^{2}\left[T^{k}\left(C_{\mathrm{nsr}}\right) \cdot T^{k}(\mathbf{N}, \mathbf{I})+T^{k}(\mathbf{N}, \mathbf{I}) \cdot T^{k}\left(C_{\mathrm{nsr}}\right)\right]$,

where $C_{\mathrm{nsr}}$ is a spin-rotation coupling tensor. Both ${ }^{225} \mathrm{Ra}$ and the hydrogen nuclei in the ortho-NSI contribute to the nuclear spin-rotation interaction. The former produces a diagonal shift of $\sim 4 \mathrm{kHz}$ for $\mathbf{N} \cdot \mathbf{I}_{R}$ interactions, while the latter produces both a diagonal shift of $\sim 15 \mathrm{kHz}$ for $\mathbf{N} \cdot \mathbf{I}_{H}$ interactions and $\sim 300 \mathrm{~Hz} K$ doubling between states differing by $\Delta K=2$.

Measurement.-Since the energy shift from the Schiff moment is proportional to the projection of the radium spin onto the molecular axis $\left(\mathbf{I}_{M} \cdot \hat{\mathbf{n}}\right)$, different hyperfine states have different Schiff moment sensitivities. In the fully decoupled limit $(\geq 1 \mathrm{~V} / \mathrm{cm})$, the value of $\left\langle\mathbf{I}_{M} \cdot \hat{\mathbf{n}}\right\rangle$ is $K \cdot m_{N} \cdot m_{I M} / 2$, where the prefactor arises because we do not mix rotational states beyond $N=1$ and thus the maximum projection of the internuclear axis on the lab frame is 1/2 (see Fig. 3, and Table S4 of the Supplemental Material [61]). There are therefore three distinct classes of states, with positive, zero, and negative Schiff energy shifts. Even in the intermediate regime when a molecule is polarized, but the spins are not decoupled $\left(\sim 50 \mathrm{mV} \mathrm{cm}^{-1}\right)$, we still find stretched states that maximally project the radium spin onto the molecular axis.

The measurement manifold is naturally populated at cold temperatures, with $\sim 1 \%$ of molecules occupying the $N=|K|=1$ manifold at $4 \mathrm{~K}$. This yield could be increased via state-controlled reactions of $\mathrm{Ra}^{+}$and methanol [96].
State preparation is possible through state-selective dissociation [36] or nondestructive quantum logic [97,98] via cotrapped $\mathrm{Ra}^{+}$[38].

Two schemes can be used for performing the EDM measurement. In the spin interferometery (SI) method [22], the molecule precesses between states of different Schiff sensitivity. After a time $\tau$, the phase $\phi=\left(\omega_{B}+\omega_{T P}\right) \tau$ is extracted via projective measurements, where $\omega_{B} \propto$ $g_{\text {eff }} \mu_{N}\left|B_{0}\right| / \hbar$ is the Larmor precession frequency and $\omega_{T P} \propto \Delta H_{\mathrm{sm}} / \hbar$ is the contribution from the differential Schiff moment between the two states. A proposed alternative approach, known as the clock-transition (CT) method [99], uses time-dependent electromagnetic fields to drive transitions between different hyperfine "clock" states. The $T, P$-violating interaction is then extracted from phase-dependent shifts to the measured Rabi oscillations. This technique, which is readily adapted to an ion trap, benefits from a simpler state preparation scheme and better robustness to electromagnetic noise.

In the polarized limit, the rotational manifold contains many hyperfine states for driving the SI or CT measurement scheme. Each state has different effective $g$-factors and Schiff sensitivity (see Fig. 3). Performing an EDM measurement with pairs of states which have unique differential magnetic sensitivities enables one to adjust the Larmor precession without changing the applied $B$ field. In addition, there are multiple pairs of near-degenerate states of opposite $m_{F}$ with the same Schiff moment sensitivity, but different magnetic moments. These add to the set of valuable systematic checks.

BSM sensitivity.-Calculations of the Schiff moment of ${ }^{225} \mathrm{Ra}$ nuclei have been performed in the framework of $T$, $P$-violating pion exchange between nucleons [15], yielding parametrizations of $\mathbf{S}\left({ }^{225} \mathrm{Ra}\right)$ in terms of the QCD $\bar{\theta}$ angle given by $\left|\mathbf{S}\left({ }^{225} \mathrm{Ra}\right)\right|=1.0 \bar{\theta} e \mathrm{fm}^{3}$ [100]. The electrostatic interactions generated by the Schiff moment leads to an effective $T, \quad P$-violating shift $H_{\mathrm{sm}}=W_{s}(\mathbf{I} \cdot \hat{\mathbf{n}})|\mathbf{S}| /|\mathbf{I}|$. The species-dependent coupling constant $W_{s}$, which is an electron-nuclear contact term, has been calculated to be 45192 atomic units in $\mathrm{RaO}$ [30] and is estimated to be slightly smaller ( 30000 a.u.) for $\mathrm{RaOH}^{+}$ (a.u. $\equiv e / 4 \pi \epsilon_{0} a_{0}^{4}$ ), where the larger ligand is assumed to reduce both electron density around $\mathrm{Ra}$ and the magnitude of $W_{s}$ [28].

To illustrate the power of a Schiff moment measurement on ${ }^{225} \mathrm{RaOCH}_{3}^{+}$, we can combine the QCD parametrization of $\mathbf{S}\left({ }^{225} \mathrm{Ra}\right)$ with the estimate $W_{s}\left({ }^{225} \mathrm{RaOCH}_{3}^{+}\right) \approx$ 30000 a.u. to calculate the averaging time needed to reach a new model-dependent limit on QCD $\bar{\theta}$. We assume a single trapped ${ }^{225} \mathrm{RaOCH}_{3}^{+}$ion with $5 \mathrm{~s}$ coherence time limited by black-body pumping at $300 \mathrm{~K}$, which provides a frequency sensitivity of $\delta \omega=7.5 \mathrm{mrad} \mathrm{s}^{-1} / \sqrt{\mathrm{h}}$. Spinprecession measurements with this setup would reach a statistical sensitivity $\bar{\theta}<10^{-10}$ with 2 weeks of data taking. Trapping multiple ions and improving the coherence time 
through cryogenic cooling would result in even higher sensitivity.

Conclusion and outlook.-We have considered trapped ${ }^{225} \mathrm{RaOCH}_{3}^{+}$as a sensitive platform to search for a nuclear Schiff moment and probe new $C P$-violating physics in the octopole-deformed $\mathrm{Ra}$ nucleus. While our theoretical calculations do not replace the need for detailed spectroscopic studies on this particular species, they do illustrate advantageous structures that are quite general for symmetric and asymmetric top molecules [94,101,102]. These include polyatomic molecules containing other heavy nuclei such as $\mathrm{Ba}[103], \mathrm{Lu}[39,104], \mathrm{Pa}$ [105], and $\mathrm{U}$ [106] (as opposed to Ra), or a different, potentially chiral, ligand (as opposed to methoxy).

The rich internal complexity of these molecules, furthermore, makes them attractive for a broad range of studies not limited to Schiff moment measurements [1]. Much of the discussion in this Letter, for instance, is directly applicable to searches for the electron EDM or nuclear magnetic quadrupole moments [34,39]. Opposite parity states with diverse, tunable splittings are generically useful for precision measurement of electroweak physics, such as nuclear spin-dependent parity violation [107] and oscillating symmetry violations from interactions with axionlike fields [108-110], and the sources that generate the splittings can themselves be sensitive to variations of fundamental constants $[111,112]$.

We are grateful for extensive assistance from Anastasia Borschevsky and Y. A. Chamorro Mena with the $a b$ initio calculations, and to Ben Augenbraun, Mingyu Fan, Alex Frenett, Arian Jadbabaie, Andrew Jayich, Ivan Kozyryev, Zack Lasner, and Tim Steimle for helpful discussions and feedback. This research was supported by a NIST Precision Measurement Grant (60NANB18D253), the Gordon and Betty Moore Foundation (7947), and the Alfred P. Sloan Foundation (G-2019-12502). Computations in this Letter were performed on the Caltech High Performance Cluster.

*phelanyu@ caltech.edu

hutzler@caltech.edu

[1] M. S. Safronova, D. Budker, D. Demille, D. F. J. Kimball, A. Derevianko, and C. W. Clark, Rev. Mod. Phys. 90, 025008 (2018).

[2] T. E. Chupp, P. Fierlinger, M. J. Ramsey-Musolf, and J. T. Singh, Rev. Mod. Phys. 91, 015001 (2019).

[3] M. Dine and A. Kusenko, Rev. Mod. Phys. 76, 1 (2003).

[4] H.-Y. Cheng, Phys. Rep. 158, 1 (1988).

[5] N. Yamanaka, B. K. Sahoo, N. Yoshinaga, T. Sato, K. Asahi, and B. P. Das, Eur. Phys. J. A 53, 54 (2017).

[6] J. Engel, M. J. Ramsey-Musolf, and U. van Kolck, Prog. Part. Nucl. Phys. 71, 21 (2013).

[7] J. S. M. Ginges and V. V. Flambaum, Phys. Rep. 397, 63 (2004).

[8] L. I. Schiff, Phys. Rev. 132, 2194 (1963).
[9] O. P. Sushkov, V. V. Flambaum, and I. B. Khriplovich, Zh. Eksp. Teor. Fiz. 87, 1521 (1984).

[10] V. V. Flambaum and J. S. M. Ginges, Phys. Rev. A 65, 032113 (2002).

[11] R. H. Parker, M. R. Dietrich, M. R. Kalita, N. D. Lemke, K. G. Bailey, M. Bishof, J. P. Greene, R. J. Holt, W. Korsch, Z. T. Lu, P. Mueller, T. P. O'Connor, and J. T. Singh, Phys. Rev. Lett. 114, 233002 (2015).

[12] M. Bishof, R. H. Parker, K. G. Bailey, J. P. Greene, R. J. Holt, M. R. Kalita, W. Korsch, N. D. Lemke, Z.-T. Lu, P. Mueller, T. P. O'Connor, J. T. Singh, and M. R. Dietrich, Phys. Rev. C 94, 025501 (2016).

[13] L. P. Gaffney et al., Nature (London) 497, 199 (2013).

[14] N. Auerbach, V. V. Flambaum, and V. Spevak, Phys. Rev. Lett. 76, 4316 (1996).

[15] J. Dobaczewski and J. Engel, Phys. Rev. Lett. 94, 232502 (2005).

[16] J. Dobaczewski, J. Engel, M. Kortelainen, and P. Becker, Phys. Rev. Lett. 121, 232501 (2018).

[17] N. Sachdeva et al., Phys. Rev. Lett. 123, 143003 (2019).

[18] F. Allmendinger, I. Engin, W. Heil, S. Karpuk, H.-J. Krause, B. Niederlander, A. Offenhausser, M. Repetto, U. Schmidt, and S. Zimmer, Phys. Rev. A 100, 022505 (2019).

[19] B. Graner, Y. Chen, E. G. Lindahl, and B. R. Heckel, Phys. Rev. Lett. 116, 161601 (2016).

[20] P. A. Butler et al., Nat. Commun. 10, 2473 (2019).

[21] J. J. Hudson, D. M. Kara, I. J. Smallman, B. E. Sauer, M. R. Tarbutt, and E. A. Hinds, Nature (London) 473, 493 (2011).

[22] W. B. Cairncross, D. N. Gresh, M. Grau, K. C. Cossel, T. S. Roussy, Y. Ni, Y. Zhou, J. Ye, and E. A. Cornell, Phys. Rev. Lett. 119, 153001 (2017).

[23] V. Andreev, D. G. Ang, D. DeMille, J. M. Doyle, G. Gabrielse, J. Haefner, N. R. Hutzler, Z. Lasner, C. Meisenhelder, B. R. O'Leary, C. D. Panda, A. D. West, E. P. West, and X. Wu, Nature (London) 562, 355 (2018).

[24] D. Cho, K. Sangster, and E. A. Hinds, Phys. Rev. A 44, 2783 (1991).

[25] L. R. Hunter, S. K. Peck, A. S. Greenspon, S. S. Alam, and D. DeMille, Phys. Rev. A 85, 012511 (2012).

[26] O. Grasdijk, O. Timgren, J. Kastelic, T. Wright, S. Lamoreaux, D. DeMille, K. Wenz, M. Aitken, T. Zelevinsky, T. Winick, and D. Kawall, arXiv:2010.01451.

[27] V. A. Dzuba, V. V. Flambaum, J. S. M. Ginges, and M. G. Kozlov, Phys. Rev. A 66, 012111 (2002).

[28] V. V. Flambaum, Phys. Rev. C 99, 035501 (2019).

[29] L. V. Skripnikov, N. S. Mosyagin, A. V. Titov, and V. V. Flambaum, Phys. Chem. Chem. Phys. 22, 18374 (2020).

[30] A. D. Kudashov, A. N. Petrov, L. V. Skripnikov, N. S. Mosyagin, A. V. Titov, and V. V. Flambaum, Phys. Rev. A 87, 020102(R) (2013).

[31] T. A. Isaev, A. V. Zaitsevskii, and E. Eliav, J. Phys. B 50, 225101 (2017).

[32] R. F. G. Ruiz et al., Nature (London) 581, 396 (2020).

[33] V. V. Flambaum and V. A. Dzuba, Phys. Rev. A 101, 042504 (2020).

[34] I. Kozyryev and N. R. Hutzler, Phys. Rev. Lett. 119, 133002 (2017). 
[35] A. V. Kudrin, A. Zaitsevskii, T. A. Isaev, D. E. Maison, and L. V. Skripnikov, Atoms 7, 62 (2019).

[36] Y. Zhou, Y. Shagam, W. B. Cairncross, K. B. Ng, T. S. Roussy, T. Grogan, K. Boyce, A. Vigil, M. Pettine, T. Zelevinsky, J. Ye, and E. A. Cornell, Phys. Rev. Lett. 124, 053201 (2020).

[37] M. Fan, C. A. Holliman, X. Shi, H. Zhang, M. W. Straus, X. Li, S. W. Buechele, and A. M. Jayich, preceding Letter, Phys. Rev. Lett. 126, 023002 (2021).

[38] M. Fan, C. A. Holliman, A. L. Wang, and A. M. Jayich, Phys. Rev. Lett. 122, 223001 (2019).

[39] D. E. Maison, L. V. Skripnikov, V. V. Flambaum, and M. Grau, arXiv:2006.03848.

[40] D. Mitra, N. B. Vilas, C. Hallas, L. Anderegg, B. L. Augenbraun, L. Baum, C. Miller, S. Raval, and J. M. Doyle, Science 369, 1366 (2020).

[41] I. Kozyryev, T. C. Steimle, P. Yu, D.-T. Nguyen, and J. M. Doyle, New J. Phys. 21, 052002 (2019).

[42] B. Z. Li, J. Xin, and L. M. Ziurys, Chem. Phys. Lett. 280, 513 (1997).

[43] B. O. Roos, R. Lindh, P.-A. Malmqvist, V. Veryazov, and P.-O. Widmark, J. Phys. Chem. A 108, 2851 (2004).

[44] B. O. Roos, V. Veryazov, and P.-O. Widmark, Theor. Chem. Accounts 111, 345 (2004).

[45] P.-O. Widmark, P.-A. Malmqvist, and B. O. Roos, Theor. Chim. Acta 77, 291 (1990).

[46] B. P. Pritchard, D. Altarawy, B. Didier, T. D. Gibson, and T. L. Windus, J. Chem. Inf. Model. 59, 4814 (2019).

[47] K. L. Schuchardt, B. T. Didier, T. Elsethagen, L. Sun, V. Gurumoorthi, J. Chase, J. Li, and T. L. Windus, J. Chem. Inf. Model. 47, 1045 (2007).

[48] D. Feller, J. Comput. Chem. 17, 1571 (1996).

[49] J. F. Stanton et al., CFOUR, Coupled-Cluster techniques for Computational Chemistry, a quantum-chemical program package. For the current version, see http://www .cfour.de.

[50] D. A. Matthews, L. Cheng, M. E. Harding, F. Lipparini, S. Stopkowicz, T.-C. Jagau, P. G. Szalay, J. Gauss, and J. F. Stanton, J. Chem. Phys. 152, 214108 (2020).

[51] M. E. Harding, T. Metzroth, J. Gauss, and A. A. Auer, J. Chem. Theory Comput. 4, 64 (2008).

[52] K. G. Dyall, J. Chem. Phys. 106, 9618 (1997).

[53] W. Liu and D. Peng, J. Chem. Phys. 131, 031104 (2009).

[54] L. Cheng and J. Gauss, J. Chem. Phys. 135, 084114 (2011).

[55] I. A. Aucar, S. S. Gmez, M. C. R. de Aza, and C. G. Giribet, J. Chem. Phys. 136, 204119 (2012).

[56] I. A. Aucar, S. S. Gmez, J. I. Melo, C. C. Giribet, and M. C. R. de Aza, J. Chem. Phys. 138, 134107 (2013).

[57] I. A. Aucar, S. S. Gomez, C. G. Giribet, and M. C. R. de Aza, J. Chem. Phys. 141, 194103 (2014).

[58] A. S.P. Gomes et al., DIRAC, a relativistic ab initio electronic structure program, Release DIRAC19 (2019), https://doi.org/10.5281/zenodo.3572669, see also http:// www.diracprogram.org.

[59] K. G. Dyall, J. Phys. Chem. A 113, 12638 (2009).

[60] P. J. Stephens, F. J. Devlin, C. F. Chabalowski, and M. J. Frisch, J. Phys. Chem. 98, 11623 (1994).

[61] See Supplemental Material at http://link.aps.org/ supplemental/10.1103/PhysRevLett.126.023003 for a dis- cussion of the effective Hamiltonian and $a b$ initio parameters, which includes Refs. [62-84].

[62] K. Raghavachari, G. W. Trucks, J. A. Pople, and M. Head-Gordon, Chem. Phys. Lett. 157, 479 (1989).

[63] R. J. Bartlett, J. D. Watts, S. A. Kucharski, and J. Noga, Chem. Phys. Lett. 165, 513 (1990).

[64] P. Crozet, A. J. Ross, C. Linton, A. G. Adam, W. S. Hopkins, and R. J. L. Roy, J. Mol. Spectrosc. 229, 224 (2005).

[65] L. C. O'Brien, C. R. Brazier, and P. F. Bernath, J. Mol. Spectrosc. 130, 33 (1988).

[66] D. Bykov, T. Petrenko, R. Izsk, S. Kossmann, U. Becker, E. Valeev, and F. Neese, Mol. Phys. 113, 1961 (2015).

[67] F. Neese, WIREs Comput. Mol. Sci. 2, 73 (2012).

[68] T. H. Dunning, J. Chem. Phys. 90, 1007 (1989).

[69] J. G. Hill and K. A. Peterson, J. Chem. Phys. 147, 244106 (2017).

[70] I. S. Lim, H. Stoll, and P. Schwerdtfeger, J. Chem. Phys. 124, 034107 (2006).

[71] S. S. Kondov, C.-H. Lee, K. H. Leung, C. Liedl, I. Majewska, R. Moszynski, and T. Zelevinsky, Nat. Phys. 15, 1118 (2019).

[72] I. Manai, R. Horchani, H. Lignier, P. Pillet, D. Comparat, A. Fioretti, and M. Allegrini, Phys. Rev. Lett. 109, 183001 (2012).

[73] T. Shimasaki, M. Bellos, C. D. Bruzewicz, Z. Lasner, and D. DeMille, Phys. Rev. A 91, 021401(R) (2015).

[74] P. F. Staanum, K. Hjbjerre, P. S. Skyt, A. K. Hansen, and M. Drewsen, Nat. Phys. 6, 271 (2010).

[75] N. B. Khanyile, G. Shu, and K. R. Brown, Nat. Commun. 6, 7825 (2015).

[76] A. E. Leanhardt, J. L. Bohn, H. Loh, P. Maletinsky, E. R. Meyer, L. C. Sinclair, R. P. Stutz, and E. A. Cornell, J. Mol. Spectrosc. 270, 1 (2011).

[77] N. Vanhaecke and O. Dulieu, Mol. Phys. 105, 1723 (2007).

[78] S. C. Wofsy, J. S. Muenter, and W. Klemperer, J. Chem. Phys. 53, 4005 (1970).

[79] M. R. Aliev and J. K. G. Watson, J. Mol. Spectrosc. 61, 29 (1976).

[80] K. Kawaguchi, Can. J. Phys. 79, 449 (2001).

[81] Y. Endo, C. Yamada, S. Saito, and E. Hirota, J. Chem. Phys. 77, 3376 (1982).

[82] Y. Endo, S. Saito, and E. Hirota, J. Chem. Phys. 81, 122 (1984).

[83] C. Adamo and V. Barone, J. Chem. Phys. 110, 6158 (1999).

[84] T. Chupp and M. Ramsey-Musolf, Phys. Rev. C 91, 035502 (2015).

[85] E. Arnold, W. Borchers, M. Carre, H. T. Duong, P. Juncar, J. Lerme, S. Liberman, W. Neu, R. Neugart, E. W. Otten, M. Pellarin, J. Pinard, G. Ulm, J. L. Vialle, and K. Wendt, Phys. Rev. Lett. 59, 771 (1987).

[86] B. Singh, Nucl. Data Sheets 106, 601 (2005).

[87] K.-i. C. Namiki, J.S. Robinson, and T. C. Steimle, J. Chem. Phys. 109, 5283 (1998).

[88] W. Klemperer, K. K. Lehmann, J. K. G. Watson, and S. C. Wofsy, J. Phys. Chem. 97, 2413 (1993).

[89] R. J. Butcher, C. Chardonnet, and C. J. Borde, Phys. Rev. Lett. 70, 2698 (1993).

[90] C. Schneider, M. Enderlein, T. Huber, and T. Schaetz, Nat. Photonics 4, 772 (2010). 
[91] J. T. Hougen, J. Mol. Spectrosc. 81, 73 (1980).

[92] J. M. Brown and A. Carrington, Rotational Spectroscopy of Diatomic Molecules (Cambridge University Press, Cambridge, England, 2003).

[93] G. R. Gunther-Mohr, C. H. Townes, and J. H. Van Vleck, Phys. Rev. 94, 1191 (1954).

[94] M. L. Wall, K. Maeda, and L. D. Carr, Ann. Phys. (Amsterdam) 525, 845 (2013).

[95] E. Hirota, High-Resolution Spectroscopy of Transient Molecules (Springer-Verlag, Berlin, 1985), Vol. 40.

[96] M.-G. Hu, Y. Liu, M. A. Nichols, L. Zhu, G. Qumner, O. Dulieu, and K.-K. Ni, arXiv:2005.10820.

[97] D. Patterson, Phys. Rev. A 97, 033403 (2018).

[98] C.-w. Chou, C. Kurz, D. B. Hume, P. N. Plessow, D. R. Leibrandt, and D. Leibfried, Nature (London) 545, 203 (2017).

[99] M. Verma, A. M. Jayich, and A. C. Vutha, Phys. Rev. Lett. 125, 153201 (2020).

[100] V. V. Flambaum and H. Feldmeier, Phys. Rev. C 101, 015502 (2020).

[101] B. L. Augenbraun, J. M. Doyle, T. Zelevinsky, and I. Kozyryev, Phys. Rev. X 10, 031022 (2020).
[102] P. Yu, L. W. Cheuk, I. Kozyryev, and J. M. Doyle, New J. Phys. 21, 093049 (2019).

[103] P. Puri, M. Mills, C. Schneider, I. Simbotin, J. A. Montgomery, R. Ct, A. G. Suits, and E. R. Hudson, Science 357, 1370 (2017).

[104] W. Lu and S. Yang, J. Phys. Chem. A 102, 825 (1998).

[105] V. V. Flambaum, Phys. Rev. A 77, 024501 (2008).

[106] M. Wormit, M. Olejniczak, A.-L. Deppenmeier, A. Borschevsky, T. Saue, and P. Schwerdtfeger, Phys. Chem. Chem. Phys. 16, 17043 (2014).

[107] E. B. Norrgard, D. S. Barker, S. Eckel, J. A. Fedchak, N. N. Klimov, and J. Scherschligt, Commun. Phys. 2, 77 (2019).

[108] P. W. Graham and S. Rajendran, Phys. Rev. D 84, 055013 (2011).

[109] Y. V. Stadnik and V. V. Flambaum, Phys. Rev. D 89, 043522 (2014).

[110] V. V. Flambaum and H. B. T. Tan, Phys. Rev. D 100, 111301(R) (2019).

[111] P. Jansen, H. L. Bethlem, and W. Ubachs, J. Chem. Phys. 140, 010901 (2014).

[112] I. Kozyryev, Z. Lasner, and J. M. Doyle, arXiv:1805.08185. 